\title{
Short communication: Longitudinal study of quarter-level somatic cell responses after naturally occurring, nonsevere clinical mastitis diagnosed as culture negative, or caused by Escherichia coli or Klebsiella pneumoniae, and randomly assigned to a no-treatment group or to receive intramammary ceftiofur
}

\author{
M. J. Fuenzalida ${ }^{1}$ and P. L. Ruegg ${ }^{2 *}$ (1) \\ ${ }^{1}$ Department of Dairy Science, University of Wisconsin, Madison 53706 \\ ${ }^{2}$ Department of Animal Science, Michigan State University, East Lansing 48864
}

\section{ABSTRACT}

The objective of this study was to describe weekly quarter-level somatic cell count (QSCC) after occurrence of nonsevere clinical mastitis (CM) that was diagnosed as culture negative, or caused by Escherichia coli or Klebsiella pneumoniae. All cases occurred in cows enrolled in negatively, controlled randomized clinical trials. We hypothesized that after occurrence of CM, QSCC patterns would vary among etiologies and this effect would not be mitigated by treatment using intramammary (IMM) ceftiofur. Data from two previously published randomized clinical trials performed on 3 Wisconsin dairy farms were used. Only cases confirmed as culture negative (NG) or E. coli or Kleb. pneumoniae (GRAMNEG) were used for analysis. In NG, cows were assigned to no antimicrobial treatment (negative control, $\mathrm{n}=44)$ or $5 \mathrm{~d}$ of once daily IMM $(\mathrm{n}=41)$ infusions with an approved product containing ceftiofur hydrochloride. In GRAMNEG, cows were assigned to IMM treatment with the same ceftiofur product for 2 different durations ( $2 \mathrm{~d}, \mathrm{n}=36$; or $8 \mathrm{~d}, \mathrm{n}=38$ ) or no antimicrobial treatment (negative control, $\mathrm{n}=36$ ). For quarters enrolled in NG, no significant differences were identified for weekly QSCC between quarters in the treated or negative control groups $\left(5.4 \log _{10} \mathrm{SCC}\right.$ for both groups). For quarters enrolled in GRAMNEG, no significant differences were identified for QSCC between quarters that received the 2 - $\mathrm{d}\left(6.2 \log _{10} \mathrm{SCC}\right)$ or 8 -d (6.3 $\left.\log _{10} \mathrm{SCC}\right)$ IMM treatment or were in the negative control group (6.0 $\left.\log _{10} \mathrm{SCC}\right)$. At the pathogen level, regardless of treatment, QSCC varied among pathogens and $\log _{10} \mathrm{SCC}$ were 5.4 (culture negative), 5.8 (E. coli), and 6.2 (Kleb. pneumoniae). Patterns of QSCC of CM

Received December 20, 2018.

Accepted July 16, 2019.

*Corresponding author: plruegg@msu.edu diagnosed as culture negative and E. coli were similar in magnitude and time to resolution of the inflammatory response. In conclusion, as compared to CM diagnosed as culture negative or caused by E. coli, CM caused by Kleb. pneumoniae was associated with poorer outcomes. Regardless of IMM ceftiofur treatment, the immune response of the cow resulted in rapid reduction of SCC of quarters diagnosed as culture negative and quarters with $\mathrm{CM}$ caused by E. coli. In contrast, the SCC remained elevated for quarters with CM caused by Kleb. pneumoniae and a greater proportion of those cases remained chronically infected.

Key words: quarter somatic cell count, Escherichia coli, Klebsiella pneumoniae, mastitis

\section{Short Communication}

Mastitis is defined as inflammation of the mammary gland due to an IMI and is the most frequently diagnosed disease of dairy cows (USDA APHIS, 2014). Clinical mastitis (CM) is often categorized based on magnitude of the inflammatory response after IMI and approximately $85 \%$ of cases are categorized as mild (abnormal milk) or moderate (abnormal milk and abnormal udder), which are together referred to as nonsevere (Oliveira et al., 2013). The interaction between pathogen and host factors dictates the immune response to a specific IMI (Schukken et al., 2011b). Based on composite SCC values from DHI data, IMI caused by Escherichia coli generally result in short duration IMI that is characterized by a rapid increase in SCC that quickly returns to normal (Todhunter et al., 1991; de Haas et al., 2002). Based on monthly composite DHI SCC, patterns of SCC for culture-negative cases of CM have been reported to be similar to those described for E. coli (de Haas et al., 2002). Many grampositive pathogens cause long-duration infection that is accompanied by consistently high monthly DHI SCC 
before, during, and after detection of a clinical case (de Haas et al., 2002). Other gram-negative pathogens such as Klebsiella spp. are also known to cause chronic and persistent IMI (Schukken et al., 2012). As SCC is one of the most important economic traits in the dairy industry, research has been focused on strategies to reduce the SCC response after IMI. We recently demonstrated that intramammary (IMM) antimicrobial treatment did not have an effect on quarter SCC (QSCC) for $\mathrm{CM}$ that was culture-negative at detection (Fuenzalida and Ruegg, 2019a), but comparison of QSCC responses among pathogens after nonsevere $\mathrm{CM}$ has not been previously described.

The inflammatory response is very similar for cases of CM caused by E. coli or diagnosed as culture negative (de Haas et al., 2002), but undesirable clinical outcomes occur less frequently for nonsevere, culture negative cases of CM (Fuenzalida and Ruegg, 2019a). As compared with CM caused by E. coli, CM caused by Klebsiella pneumoniae is associated with more detrimental consequences (Fuenzalida and Ruegg, 2019b). Descriptions of quarter-level inflammatory response after IMI diagnosed as culture-negative, or caused by E. coli and Klebsiella spp., are lacking. The objective of this short communication is to describe weekly QSCC after occurrence of nonsevere CM that was diagnosed as culture negative, or caused by E. coli or Kleb. pneumoniae. All cases occurred in cows prospectively enrolled in negatively, controlled randomized clinical (RCT). The hypothesis was that after the occurrence of CM, patterns of SCC at the quarter level would vary based on etiology and this effect would not be mitigated by IMM treatment using ceftiofur.

Data were used from 2 separate but similarly designed negatively controlled randomized clinical trials that have been previously published (Fuenzalida and Ruegg, 2019a,b). In both trials, only cases of naturally occurring CM categorized as mild (abnormal milk appearance) or moderate (abnormal milk and udder appearance) were enrolled. All herds had monthly DHI milk testing that included individual SCC data, and used computerized herd records that included mastitis events and treatments. Before the study started, farm workers were trained to collect aseptic quarter milk samples, record a standardized mastitis severity score (Pinzón-Sánchez et al., 2011), and perform on-farm culture (OFC) to identify eligible cases that were then randomized among treatment or control groups. Data were collected for $90 \mathrm{~d}$ after enrollment or until the end of lactation (follow-up period, FUP). All milk samples collected from enrolled cases were subjected to confirmatory microbiological diagnoses at the University of Wisconsin (UW) Milk Quality laboratory according to National Mastitis Council guidelines (NMC, 1999, 2017). All procedures for both trials were approved by the Animal Care and Use Committee for the College of Agriculture and Life Sciences of the UW-Madison (protocol A01574-0-06-14).

Trial 1 (NG) enrolled culture-negative cases of CM that occurred between March 2015 through May 2017, and only data from cases that were confirmed as culture negative in the UW Milk Quality laboratory were used in this analysis. The NG trial was conducted using dairy cattle at the UW dairy farms. Lactating dairy cows were managed as a single herd but were occasionally moved between 2 locations. The largest location housed approximately 470 lactating cows in a sand-bedded freestall barn and the smallest facility housed approximately 66 lactating dairy cows in a tiestall barn. In both facilities, dairy cows were milked twice per day in a parlor and milking technicians used standardized milking practices that included removal and observation of foremilk. Milking technicians aseptically collected duplicate quarter-milk samples from eligible nonsevere cases of CM and performed on-farm culture to identify eligible cases (Fuenzalida and Ruegg, 2019a). After $24 \mathrm{~h}$, trained farm workers observed biplates for microbial growth. Culture negative cases were randomly assigned to either a treatment or negative control group. Quarters assigned to treatment received one daily IMM infusion with a commercially available approved product containing ceftiofur hydrochloride (Spectramast LC, Zoetis, Florham Park, NJ) for $5 \mathrm{~d}$. Quarters assigned to the negative control group did not receive any antimicrobial therapy or any other interventions. Researchers visited farms weekly to retrieve computerized cow health and performance records (DairyComp305, Valley Agricultural Software, Tulare, $\mathrm{CA}$ ), and collect duplicate quarter milk samples from enrolled quarters at 7, 14, 21, 28, 35, 42, 49, 56, 63, 70, 77 , and $84 \pm 3 \mathrm{~d}$ after enrollment. All milk samples were preserved with bronopol and transported to a commercial DHI laboratory (AgSource CRI, Verona, WI) for SCC analysis (CombiFOSS 6000, Foss Food Technology Corp., Hillerød, Denmark).

Trial 2 (GRAMNEG) enrolled gram-negative cases of CM occurring on 2 commercial Wisconsin dairy herds that had a single owner between June and December 2016 and only data from cases that were confirmed as gram-negative in the UW Milk Quality laboratory were used in this analysis. Study design, case enrollment criteria, data collection, microbiological analysis, and definitions have been previously described by Fuenzalida and Ruegg (2019b). Each of the farms contained more than 3,000 lactating dairy cows and lactating cows were housed in freestall barns containing processed manure 
solids and milked 3 times per day in rotary parlors by trained milking technicians using complete milking routine including removal and observation of foremilk. Upon detection of a nonsevere CM case, farm workers assigned a severity score and collected an aseptic milk sample that was used to perform on-farm culture (Fuenzalida and Ruegg, 2019b). After 24 h, plates were observed for microbial growth and cases categorized as gram-negative were randomly assigned to either negative control, 2-d or 8-d IMM treatment with a commercially available, approved product containing ceftiofur hydrochloride (Spectramast LC, Zoetis). Quarters assigned to the negative control group did not receive any antimicrobial therapy. Milk samples were collected aseptically from enrolled quarters at $7,14,21$, and $28 \pm$ $3 \mathrm{~d}$ after enrollment. One duplicate sample was used for further microbiological analysis and the other samples were preserved with bronopol and transported to a commercial DHI laboratory (AgSource CRI, Verona, WI) for SCC analysis. Based on microbiological results of milk samples enrolled in the study and cultured in the UW Milk Quality laboratory, only cases confirmed as E. coli or Kleb. pneumoniae were included in this study. Microbiological procedures have been previously described (Fuenzalida and Ruegg, 2019b) and identification of bacteria at species level was performed using appropriate API tests (API 20E, bioMerieux-Vitek Inc., Hazelwood, MO).

For both studies, previous subclinical mastitis (SCM) was defined at the cow level as monthly DHI SCC $>150,000$ cells per $\mathrm{mL}$ and was measured 21 to $55 \mathrm{~d}$ before detection of the enrolled CM case. New IMM infection at 14 or $28 \mathrm{~d}$ after enrollment (NG) was defined as bacterial recovery from quarter milk samples at either period (Fuenzalida and Ruegg, 2019a). Bacteriological cure (GRAMNEG) was defined as no isolation of bacteria at 14 and $21 \mathrm{~d}$ after enrollment (BC; Fuenzalida and Ruegg, 2019b).

Statistical analyses were performed using SAS version 9.4 (SAS Institute, 2011). The experimental unit was quarter. Somatic cell count data were log-transformed $\left[\log _{10} \mathrm{SCC}=\log _{10}(\mathrm{SCC} \times 1,000)\right]$ to follow normal distribution.

The effect of IMM ceftiofur treatment on weekly QSCC was assessed using repeated measures ANOVA using PROC MIXED with an unstructured covariance structure. For the NG data set, putative explanatory variables initially included experimental group, parity, season of detection of CM, severity of CM, occurrence of previous diseases, new IMM infection during followup period, etiology of new IMM infections, mastitis history (CM and SCM), average milk yield $7 \mathrm{~d}$ before case detection, DIM, and time. Experimental group was forced into the model, then each variable offered to the model was evaluated by manual bidirectional elimination until all explanatory variables had $P$-value $<0.10$; subsequently, a model was chosen based on parsimony and goodness of fit [-2 log-likelihood, Akaike information criterion (AIC), corrected AIC (AICC), Bayesian information criterion (BIC)]. After model building, remaining explanatory variables included time, experimental group (forced), previous SCM, occurrence of IMI during FUP, and 2-way interactions. For the GRAMNEG data set, putative explanatory variables included experimental group, parity, season of detection of CM, severity of CM, CM history, BC, etiology of CM, 3 functional quarters before case detection, SCM history, average milk yield $7 \mathrm{~d}$ before case detection, DIM, and time. Experimental group was forced into the model, then each variable offered to the model was evaluated by manual bidirectional elimination until all explanatory variables had $P$-value $<0.10$; subsequently, a model was chosen based on parsimony and goodness of fit ( -2 log-likelihood, AIC, AICC, BIC). After model building, remaining explanatory variables included time, experimental groups (forced), etiology of CM (E. coli or Kleb. pneumoniae), BC, and 2- and 3 -way interactions. For the combined data set, potential explanatory variables included season of detection of CM, severity of CM, CM history, SCM history, DIM, etiology, and time. Farm was included in the model as a random factor. Experimental group was forced into the model, then each variable offered to the model was evaluated by manual bidirectional elimination until all explanatory variables had $P$-value $<0.10$; subsequently, a model was chosen based on parsimony and goodness of fit ( -2 log-likelihood, AIC, AICC, BIC). Cases were not included in analyses if they had missing data for a variable included in the models. Data sets from both trials were combined to describe QSCC at $7,14,21$, and $28 \mathrm{~d}$ after enrollment. For the combined effect of diagnosis (culture negative, E. coli, or Kleb. pneumoniae), the model included time, diagnosis, previous SCM, severity of CM, farm (random factor), and 2-way interactions. Goodness of fit was assessed using -2 log-likelihood, AIC, AICC, and BIC of PROC MIXED. Adjusted means of weekly QSCC were generated using LSMEANS option via PROC MIXED.

Of 121 cases enrolled in NG based on results of OFC, $70 \%$ were used in this analysis based on confirmatory microbiological results from UW Milk Quality laboratory. Cases used in this study were distributed between the negative control group $(\mathrm{n}=44)$ and the antimicrobial treatment group $(\mathrm{n}=41)$. Cases were not included due to contamination $(50 \%, \mathrm{n}=18)$ or microbial growth (50\%). Further description of agreement between OFC 
and UW Milk Quality laboratory has been described elsewhere (Fuenzalida and Ruegg, 2019a). For NG, during the 90-d FUP, LSM of weekly QSCC were $5.4 \pm 0.1$ $\log _{10} \mathrm{SCC}$ and were not associated with treatment $(P=$ 0.678 ; Figure 1). Time $(P=0.013)$, previous SCM $(P<$ $0.001)$, and occurrence of IMI during FUP $(P=0.011)$ were all associated with weekly QSCC. The interaction between treatment and time was not associated with weekly QSCC $(P=0.498)$. Weekly QSCC were greater in cows with previous SCM $\left(5.7 \pm 0.1 \log _{10} \mathrm{SCC}\right)$ compared with cows with no previous SCM history $(5.2 \pm$ $\left.0.1 \log _{10} \mathrm{SCC}\right)$. Cases that experienced IMI during the FUP had greater QSCC $\left(5.6 \pm 0.1 \log _{10} \mathrm{SCC}\right)$ compared with cases not experiencing IMI $\left(5.3 \pm 0.1 \log _{10} \mathrm{SCC}\right)$.

Of 168 cases enrolled in GRAMNEG based on results of OFC, $65 \%$ were used in this analysis based on result of microbiological tests performed at the UW Milk Quality laboratory. Cases caused by E. coli used in this study were distributed between the negative control group ( $\mathrm{n}=19)$, the 2 -d IMM ceftiofur group $(\mathrm{n}=18)$, and the 8-d IMM ceftiofur group $(\mathrm{n}=19)$. Cases caused by Kleb. pneumoniae used in this study were distributed between the negative control group (n $=17)$, the 2 -d IMM ceftiofur group $(\mathrm{n}=18)$, and the 8-d IMM ceftiofur group $(\mathrm{n}=19)$. Cases not included were contaminated $(41 \%, \mathrm{n}=24)$, missing $(9 \%, \mathrm{n}=5)$, culture negative $(34 \%, \mathrm{n}=20)$, or had gram-positive microbial growth $(16 \%, \mathrm{n}=9)$. Further description of agreement between OFC and UW Milk Quality laboratory has been described elsewhere (Fuenzalida and Ruegg, 2019b).

For GRAMNEG, LSM QSCC during the FUP were $6.2 \pm 0.1$ (negative control), $6.3 \pm 0.1$ (2-d IMM ceftiofur), and $6.0 \pm 0.1 \log _{10} \mathrm{SCC}$ (8-d IMM ceftiofur), and experimental group was not associated with QSCC $(P$ $=0.111)$. Time $(P<0.001), \mathrm{BC}(P=0.025)$, pathogen (E. coli or Kleb. pneumoniae; $P=0.001$ ), and interaction between pathogen and time $(P<0.001)$ were all associated with weekly QSCC. Weekly QSCC were greater in cows experiencing CM caused by Kleb. pneumoniae (6.4 $\pm 0.1 \log _{10} \mathrm{SCC}$ ) compared with cases caused by $E$. coli $\left(5.9 \pm 0.1 \log _{10} \mathrm{SCC}\right.$; Figure 2a). Three-way interactions between experimental groups, pathogen, and time $(P=$ 0.960 ) were not associated with weekly QSCC (Figure $2 \mathrm{~b}, \mathrm{c})$. Cases that experienced BC $\left(6.0 \pm 0.1 \log _{10} \mathrm{SCC}\right)$ had lower QSCC compared with cases that failed to BC $\left(6.3 \pm 0.1 \log _{10} \mathrm{SCC}\right)$.

In the combined data set, QSCC LSM at 7, 14, 21, and $28 \mathrm{~d}$ after enrollment were $5.4 \pm 0.1,5.8 \pm 0.1$, and $6.2 \pm 0.1 \log _{10} \mathrm{SCC}$ for nonsevere $\mathrm{CM}$ diagnosed as culture negative, or caused by E. coli and Kleb. pneumoniae, respectively (Figure 3). Pathogen $(P<$ 0.001 ; Figure 3$)$, time $(P<0.001)$, previous $\operatorname{SCM}(P=$

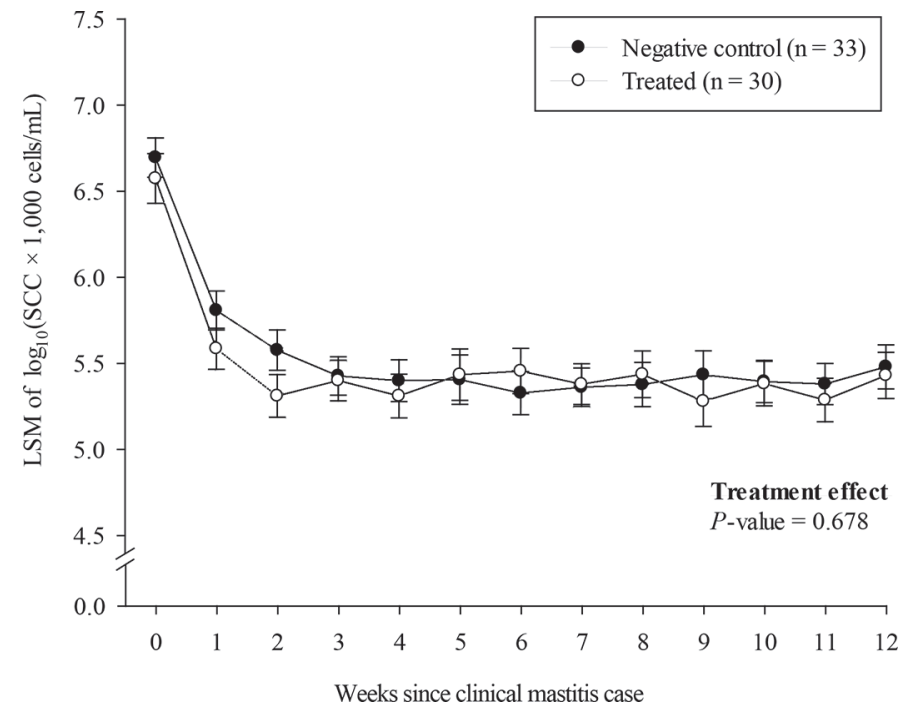

Figure 1. Least squares means of weekly quarter SCC for culture negative cases of clinical mastitis by experimental groups (treated vs. negative control). Treated cases received 5-d intramammary ceftiofur. Negative control cases received no intramammary antimicrobial or any other intervention. Repeated measures analysis included the effect of treatment, time, previous subclinical mastitis, IMI during follow-up period, and 2-way interactions. The SEM are depicted for LSM of weekly quarter SCC.

$0.005)$, and severity of $\mathrm{CM}(P=0.017)$ were all associated with weekly QSCC, and the interaction between type of pathogen and time approached significance $(P$ $=0.074$ ). Differences of LSM of weekly QSCC between $\mathrm{CM}$ caused by $E$. coli and Kleb. pneumoniae were -0.4 $\pm 0.1 \log _{10} \mathrm{SCC}(P=0.002)$. Differences of LSM of weekly QSCC between milk obtained from quarters affected by gram-negative bacteria and milk from culture negative quarters were $0.4 \pm 0.1$ ( $E$. coli vs. culture negative, $P<0.001$ ) and $0.8 \pm 0.1$ (Kleb. pneumoniae vs. culture negative, $P<0.001) \log _{10}$ SCC. Weekly QSCC were greater in cows with previous SCM (6.0 $\pm 0.1 \log _{10} \mathrm{SCC}$ ) compared with cows with no previous SCM history (5.7 $\left.\pm 0.1 \log _{10} \mathrm{SCC}\right)$, and in cows with moderate cases of CM $\left(5.9 \pm 0.1 \log _{10} \mathrm{SCC}\right)$ compared with cows with mild CM $\left(5.7 \pm 0.1 \log _{10} \mathrm{SCC}\right)$.

Somatic cell count is an objective measurement of the inflammatory response after IMI. In both trials, we measured the inflammatory response at the quarter level and our results reflected changes in QSCC after detection of $\mathrm{CM}$ diagnosed as culture negative, or caused by E. coli or Kleb. pneumoniae. This is the first study that describes the quarter-level SCC response among these diagnoses. The importance of studying quarter-level SCC response by etiology is to improve our understanding of the immune response of the cow and to understand better the resolution of IMI after treatment (or withholding of treatment). 


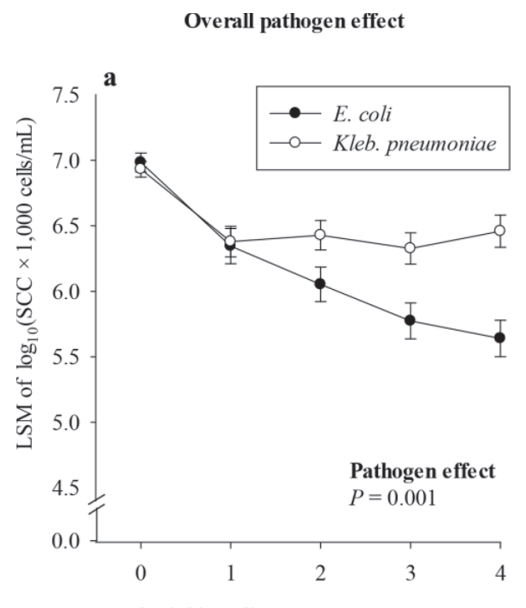

Escherichia coli by experime ntal group

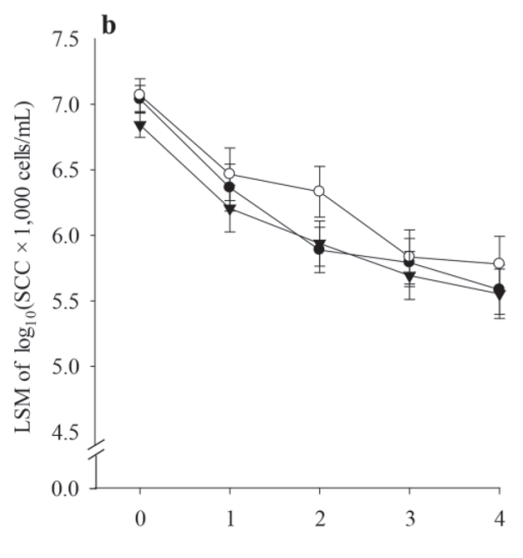

Klebsiella pneumoniae by experimental group

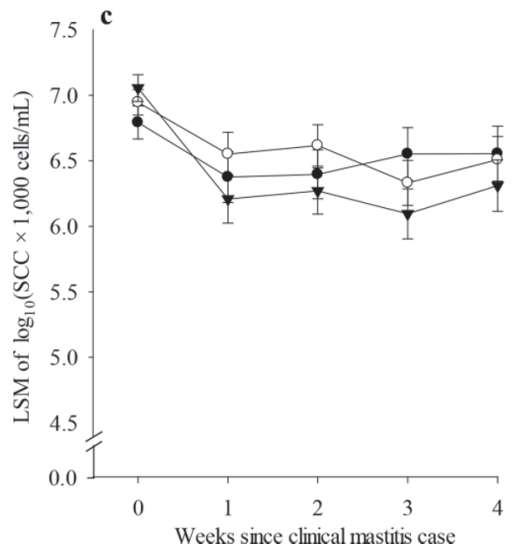

Figure 2. Least squares means of weekly quarter SCC (QSCC). Repeated measures analysis included the effect of treatments, time, gram-negative bacteria, bacteriological cure, and 2- and 3-way interactions. Curves of QSCC until $28 \pm 3 \mathrm{~d}$ after enrollment by type of gram-negative bacteria (a). Curves of QSCC until $28 \pm 3$ d after enrollment of Escherichia coli clinical mastitis (CM) by experimental group: negative control (closed circle, $\mathrm{n}=17$ ), 2-d intramammary (IMM) ceftiofur (open circle, $\mathrm{n}=17$ ), and 8-d IMM ceftiofur (closed triangle, $\mathrm{n}=18$; b). Curves of QSCC CM and Klebsiella pneumoniae (c) by experimental group: negative control (closed circle, $\mathrm{n}=14$ ), 2-d IMM ceftiofur (open circle, $\mathrm{n}=17$ ), and 8-d IMM ceftiofur (closed triangle, $\mathrm{n}=16$ ) groups. The SEM are depicted for LSM of weekly quarter SCC.
Our results demonstrated that treatment of nonsevere, culture negative CM using IMM ceftiofur did not affect weekly QSCC during the 90-d FUP. As expected, when CM was detected QSCC was high, but for culture-negative cases QSCC decreased rapidly and remained consistently low. Although a slight inflammatory response likely occurred in quarters adjacent to the affected gland (Paixao et al., 2017), it is probable that SCC of composite milk collected from affected cows would be $<200,000$ cells $/ \mathrm{mL}$ within 2 to 3 wk after detection of the enrolled case. Although some culture-negative cases are likely false-negative IMI due to sparse shedding of pathogens (Fuenzalida and Ruegg, 2019a), the positive prognosis and rapid resolution of inflammation observed for these cases indicates that for many cases, the initial inflammatory response achieved bacteriological clearance before the case was detected. We previously reported that recurrence of CM, daily milk production, and risk of culling were not associated with treatment of culture-negative, nonsevere cases of CM (Fuenzalida and Ruegg, 2019a) and these data demonstrate that the immune response of the cows was successful in elimination of most of these IMI. Risk factors associated with changes in QSCC included history of previous SCM and IMI, indicating that some of these cases were preceded by a period of SCM before detection of the clinical case. To determine duration of SCM before CM, farmers should be advised to review monthly SCC and treatment records of cows affected with nonsevere $\mathrm{CM}$ and use that data as part of the

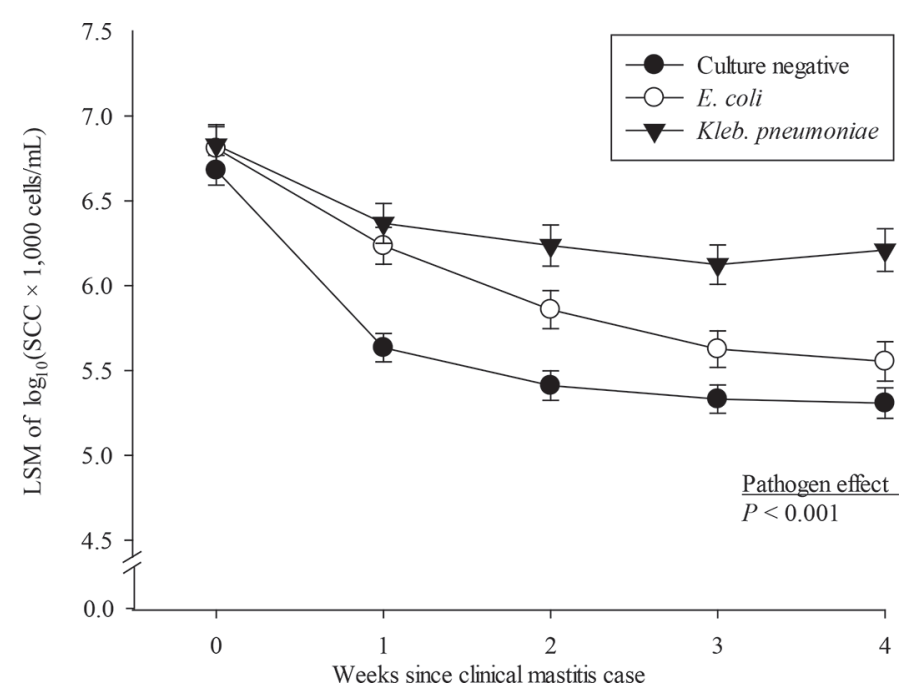

Figure 3. Least squares means of weekly quarter-SCC by pathogen; culture negative $(\mathrm{n}=64)$, Escherichia coli $(\mathrm{n}=41)$, and Klebsiella pneumoniae $(\mathrm{n}=33)$. Repeated measures analysis included the effect of type of pathogen, time, and 2-way interactions. The SEM are depicted for LSM of weekly quarter SCC. 
algorithm to determine when antimicrobial therapy may be of benefit (Ruegg, 2017).

For nonsevere CM caused by E. coli or Kleb. pneumoniae, 2-d or 8-d IMM ceftiofur treatment did not reduce weekly QSCC, although we observed large differences in QSCC based on pathogen. We previously reported that recurrence of $\mathrm{CM}$, daily milk production, and risk of culling were not associated with either 2 or $8 \mathrm{~d}$ of treatment of gram-negative, nonsevere cases of CM (Fuenzalida and Ruegg, 2019b) and the effect of antimicrobial treatment was especially insignificant for cases caused by $E$. coli. These data demonstrate that the immune response of the cows was successful in elimination of most of these IMI; however, it is possible that differences among bacterial strains could influence such results (Blum et al., 2017; Roussel et al., 2017). Regardless of treatment, when CM was caused by $E$. coli, QSCC at detection was very high but it rapidly decreased during the $28 \mathrm{~d}$ FUP, indicating rapid resolution of IMI. In contrast, when CM was caused by Kleb. pneumoniae, QSCC decreased only slightly by d 7 and then remained high without experiencing further resolution (regardless of treatment). As anticipated, $\mathrm{BC}$ was associated with QSCC after resolution of clinical signs. We previously reported that risk of $\mathrm{BC}$ varies depending among gram-negative pathogens and the immune response is very successful in elimination of $E$. coli infections but often fails to eliminate IMI caused by Kleb. pneumoniae (Fuenzalida and Ruegg, 2019b). In many instances, CM caused by Kleb. pneumoniae resulted in chronic inflammation, regardless of treatment. We previously demonstrated that $\mathrm{CM}$ caused by Kleb. pneumoniae is associated with poorer clinical outcomes (as compared with CM caused by E. coli) and these cases have a greater risk of culling and voluntary dry-off of quarters (Fuenzalida and Ruegg, 2019b).

As compared with nonsevere CM caused by E. coli, and Kleb. pneumoniae, QSCC after nonsevere, culturenegative CM returned to healthy levels earlier and remained much lower. The QSCC after CM caused by $E$. coli were less than QSCC of CM caused by Kleb. pneumoniae. Quarter SCC were lower for cases diagnosed as culture-negative as compared with cases caused by $E$. coli. Fewer negative clinical outcomes have been noted for culture-negative CM (Fuenzalida and Ruegg, 2019a) compared with cases caused by gram-negative bacteria (Fuenzalida and Ruegg, 2019b). Patterns we observed for QSCC indicated that the inflammatory response varies depending on the initial diagnosis. Our results confirmed previous findings that included cases of CM caused by gram-negative (Schukken et al., 2011a) or gram-positive (Oliver et al., 2004) bacteria and for cows randomized to culture-based or standard treatment protocols (Lago et al., 2011), but we were able to fur- ther describe how the inflammatory response occurred at the quarter level and how the response varied among gram-negative pathogens. It is important to note that these studies were conducted in a temperate climate on a limited number of farms under North American intensive farming conditions and additional work is needed to confirm the broader applicability of our results.

In conclusion, for nonsevere CM, etiology was associated with QSCC within a 28 or $90 \mathrm{~d}$ FUP. Intramammary treatment using ceftiofur did not mitigate QSCC for $\mathrm{CM}$ diagnosed as culture negative, or caused by $E$. coli or Kleb. pneumoniae. For cases of CM diagnosed as culture-negative, weekly QSCC decreased rapidly within a week of detection of the case and remained low. Similarly, for cases caused by E. coli, weekly QSCC decreased within a week and remained low afterward. In contrast, for cases caused by Kleb. pneumoniae, weekly QSCC decreased only slightly 1 wk after detection but then increased and remained high. The results of this study showed clear differences in QSCC response depending on the etiology, and for cows experiencing nonsevere mastitis, these differences may be useful when deciding on prognosis of cases.

\section{ACKNOWLEDGMENTS}

This research was supported by Hatch Act Formula Fund Grant no. WIS01799. The SpectramastLC was donated by Zoetis (Parsippany, NJ).

\section{REFERENCES}

Blum, S. E., E. D. Heller, S. Jacoby, O. Krifucks, and G. Leitner. 2017. Comparison of the immune responses associated with experimental bovine mastitis caused by different strains of Escherichia coli. J. Dairy Res. 84:190-197.

de Haas, Y., H. W. Barkema, and R. F. Veerkamp. 2002. The effect of pathogen-specific clinical mastitis on the lactation curve for somatic cell count. J. Dairy Sci. 85:1314-1323.

Fuenzalida, M. J., and P. L. Ruegg. 2019a. Negatively-controlled, randomized clinical trial to evaluate use of intramammary ceftiofur for treatment of nonsevere culture negative clinical mastitis. J. Dairy Sci. 102:3321-3338.

Fuenzalida, M. J., and P. L. Ruegg. 2019b. Negatively-controlled, randomized clinical trial to evaluate intramammary treatment of nonsevere, gram-negative clinical mastitis. J. Dairy Sci. 102:54385457.

Lago, A., S. M. Godden, R. Bey, P. L. Ruegg, and K. Leslie. 2011. The selective treatment of clinical mastitis based in on-farm culture results: I. Effects on antibiotic use, milk withholding time, and short-term clinical and bacteriological outcomes. J. Dairy Sci. 94:4441-4456.

National Mastitis Council. 1999. Laboratory Handbook on Bovine Mastitis. National Mastitis Council, Verona, WI.

National Mastitis Council. 2017. Laboratory Handbook on Bovine Mastitis. Third ed. National Mastitis Council, Verona, WI.

Oliveira, L., C. Hulland, and P. L. Ruegg. 2013. Characterization of clinical mastitis occurring in cows on 50 large dairy herds in Wisconsin. J. Dairy Sci. 96:7538-7549.

Oliver, S. P., R. A. Almeida, B. E. Gillespie, S. J. Headrick, H. H. Dowlen, D. L. Johnson, K. C. Lamar, S. T. Chester, and W. M. 
Moseley. 2004. Extended ceftiofur therapy for treatment of experimentally-induced Streptococcus uberis mastitis in lactating dairy cattle. J. Dairy Sci. 87:3322-3329.

Paixao, M. G., L. R. Abreu, R. Richert, and P. L. Ruegg. 2017. Milk composition and health status from mammary gland quarters adjacent to glands affected with naturally occurring clinical mastitis. J. Dairy Sci. 100:7522-7533.

Pinzón-Sánchez, C., V. E. Cabrera, and P. L. Ruegg. 2011. Decision tree analysis of treatment strategies for mild and moderate cases of clinical mastitis occurring in early lactation. J. Dairy Sci. 94:1873-1892.

Roussel, P., A. Porcherie, M. Reperant-Ferter, P. Cunha, C. Gitton, P. Rainard, and P. Germon. 2017. Escherichia coli mastitis strains: In vitro phenotypes and severity of infection in vivo. PLoS One 12:e0178285.

Ruegg, P. L. 2017. Practical approaches to mastitis therapy on large dairy herds. In Large Dairy Herd Management. 3rd ed. J. Hogan, ed. Am. Dairy Sci. Assoc., Champaign, IL.

SAS Institute. 2011. SAS/STAT User's Guide. Version 9.4. SAS Institute Inc., Cary, NC.

Schukken, Y., M. Chuff, P. Moroni, A. Gurjar, C. Santisteban, F. Welcome, and R. Zadoks. 2012. The "other" gram-negative bacteria in mastitis: Klebsiella, Serratia, and more. Vet. Clin. North Am. Food Anim. Pract. 28:239-256.

Schukken, Y. H., G. J. Bennett, M. J. Zurakowski, H. L. Sharkey, B. J. Rauch, M. J. Thomas, B. Ceglowski, R. L. Saltman, N. Be- lomestnykh, and R. N. Zadoks. 2011a. Randomized clinical trial to evaluate the efficacy of 5-day ceftiofur hydrochloride intramammary treatment on nonsevere gram-negative clinical mastitis. J. Dairy Sci. 94:6203-6215.

Schukken, Y. H. J., J. Günther, M. C. Fitzpatrick, L. Fontaine, O. Goetze, J. Holst, W. Leigh, H. J. Petzl, A. Schuberth, D. G. Sipka, R. Smith, J. Quesnell, R. Watts, H. Yancey, A. Zerbe, R. N. Gurjar, and H. M. Zadoks. Seyfert, and members of the Pfizer mastitis research consortium. 2011b. Host-response patterns of intramammary infections in dairy cows. Vet. Immunol. Immunopathol. 144:270-289.

Todhunter, D. A., K. L. Smith, J. S. Hogan, and P. S. Schoenberger. 1991. Gram-negative bacterial infections of the mammary gland in cows. Am. J. Vet. Res. 52:184-188.

USDA APHIS. 2014. VS:NAHMS (U.S. Department of Agriculture, Animal and Plant Health Inspection Service, Veterinary Services, National Animal Health Monitoring System). Milk quality, milking procedures, and mastitis on U.S. dairies, 2014. Accessed Apr. 2, 2018. https://www.aphis.usda.gov/animal_health/nahms/dairy/ downloads/dairy14/Dairy14_dr_Mastitis.pdf.

\section{ORCIDS}

P. L. Ruegg @ https://orcid.org/0000-0002-7211-4512 